\title{
Efficacy of corifollitropin alfa followed by recombinant follicle-stimulating hormone in a gonadotropin- releasing hormone antagonist protocol for Korean women undergoing assisted reproduction
}

\author{
Hyo Young Park' , Min Young Lee ${ }^{2}$, Hyo Young Jeong ${ }^{2}$, Yong Sook Rho², Sang Jin Song ${ }^{1}$, Bum-Chae Choi ${ }^{2}$ \\ 'Laboratory of Reproductive Medicine, Creation and Love Women's Hospital, Gwangju; '2Department of Obstetrics and Gynecology, Center for Infertility \\ and Recurrent Miscarriage, Creation and Love Women's Hospital, Gwangju, Korea
}

Objective: To evaluate the effect of a gonadotropin-releasing hormone $(\mathrm{GnRH})$ antagonist protocol using corifollitropin alfa in women undergoing assisted reproduction.

Methods: Six hundred and eighty-six in vitro fertilization-embryo transfer (IVF)/ intracytoplasmic sperm injection (ICSI) cycles were analyzed. In 113 cycles, folliculogenesis was induced with corifollitropin alfa and recombinant follicle stimulating hormone (rFSH), and premature luteinizing hormone (LH) surges were prevented with a GnRH antagonist. In the control group ( 573 cycles), premature LH surges were prevented with $\mathrm{GnRH}$ agonist injection from the midluteal phase of the preceding cycle, and ovarian stimulation was started with rFSH. The treatment duration, quality of oocytes and embryos, number of embryo transfer (ET) cancelled cycles, risk of ovarian hyperstimulation syndrome (OHSS), and the chemical pregnancy rate were evaluated in the two ovarian stimulation protocols.

Results: There were no significant differences in age and infertility factors between treatment groups. The treatment duration was shorter in the corifollitropin alfa group than in the control group. Although not statistically significant, the mean numbers of matured (86.8\% vs. $85.1 \%)$ and fertilized oocytes ( $84.2 \%$ vs. $83.1 \%$ ), good embryos (62.4\% vs. $60.3 \%)$, and chemical pregnancy rates ( $47.2 \%$ vs. $46.8 \%$ ) were slightly higher in the corifollitropin alfa group than in the control group. In contrast, rates of ET cancelled cycles and the OHSS risk were slightly lower in the corifollitropin alfa group (6.2\% and $2.7 \%$ ) than in the control group ( $8.2 \%$ and $3.5 \%)$, although these differences were also not statistically significant. Conclusion: Although no significant differences were observed, the use of corifollitropin alfa seems to offer some advantages to patients because of its short treatment duration, safety, lower ET cancellation rate and reduced risk of OHSS.

Keywords: Corifollitropin alfa; Infertility; GnRH agonist; Ovarian hyperstimulation syndrome; Recombinant follicle stimulating hormone

\section{Introduction}

Through the induction of multiple follicles, assisted reproduction

Received: Jan 13, 2015 · Revised: Feb 2, 2015 · Accepted: Apr 22, 2015 Corresponding author: Bum-Chae Choi

Department of Obstetrics and Gynecology, Center for Infertility and Recurrent Miscarriage, Creation and Love Women's Hospital, 957 Mujin-daero, Seo-gu, Gwangju 502-800, Korea

Tel: +82-62-368-1700 Fax:+82-62-368-1711 E-mail:cbcmd60@gmail.com

This is an Open Access article distributed under the terms of the Creative Commons Attribution Non-Commercial License (http://creativecommons.org/licenses/by-nc/3.0/) which permits unrestricted non-commercial use, distribution, and reproduction in any medium, provided the original work is properly cited. techniques provide the highest possibility of achieving pregnancy for infertile couples. Nevertheless, controlled ovarian stimulation (COS) has been recognized as a painstaking process for many people. It involves the maintenance of extended control over endogenous reproductive hormones and continuous dosing with exogenous hormones via abdominal injection. The long gonadotropin-releasing hormone (GnRH) agonist protocol is the most commonly used protocol. It takes approximately three weeks, effectively preventing premature LH surges and maintaining multifollicular growth. In addition, gonadotropin injections have to be administered daily by the patient due to their short elimination half-life and rapid metabolic clearance 
[1]. Thus, fertility treatment is a complex process that can be physically and emotionally demanding for infertile patients. It is also closely related to success rates. Therefore, to lessen the burden of COS, a simpler and more convenient treatment approach is needed.

Corifollitropin alfa (Elonva, NV Organon, Oss, The Netherlands) is one of the most recently introduced multifollicular stimulants. This novel molecule is a fusion product of human follicle stimulating hormone (FSH) and the carboxy-terminal peptide of human chorionic gonadotropin (hCG) [2]. Its use was developed as part of a GnRH antagonist protocol. It has the same pharmacologic activity as FSH and recombinant follicle stimulating hormone ( $\mathrm{rFSH})$, but a slower absorption rate and approximately twice the half-life length of $\mathrm{rFSH}$. Corifollitropin alfa is able to initiate and sustain follicular growth for an entire week, so a single injection can replace the first seven daily injections of gonadotropin in each ovarian stimulation treatment cycle prior to assisted reproduction $[3,4]$. Thus, corifollitropin alfa can reduce the number of injections needed for COS before in vitro fertilization (IVF) or intracytoplasmic sperm injection (ICSI) and may lower the treatment burden and stress.

The optimal dose of corifollitropin alfa has been calculated to be $100 \mu \mathrm{g}$ for patients weighing $\leq 60 \mathrm{~kg}$ and $150 \mu \mathrm{g}$ for patients weighing $>60 \mathrm{~kg}[5,6]$. Using these dosing criteria, previous studies have shown that administration of corifollitropin alfa is safe, effective, and well tolerated and that it does not result in antibody formation $[3,4,6-$ 8]. Tarlatzis et al. [9] reported no increase in the risk of ovarian hyperstimulation syndrome (OHSS) in infertile women receiving corifollitropin alfa; however, a recent systematic review suggested that there is evidence of increased ovarian response and risk of OHSS in corifollitropin alfa treatment [10]. Based on the current literature, more research is needed to assess whether a new protocol using corifollitropin alfa would benefit women undergoing infertility treatment.

In the present study, we retrospectively reviewed the records of patients treated with either corifollitropin alfa in the $\mathrm{GnRH}$ antagonist protocol or $\mathrm{rFSH}$ in the long $\mathrm{GnRH}$ agonist protocol (control group). We evaluated the treatment duration, estradiol $\left(E_{2}\right)$ concentration, total number of oocytes retrieved, quality of oocytes and embryos, number of embryo transfer (ET) cycles and ET cancelled cycles, risk of OHSS, and the chemical pregnancy rate in the two ovarian stimulation protocols.

\section{Methods}

\section{Patient population}

We retrospectively analyzed a total of 686 IVF/ICSI cycles occurring between May 2011 and May 2013. Patients were women aged 20-39 years old with a normal menstrual cycle (24-35 days) and a body mass index of $15-34 \mathrm{~kg} / \mathrm{m}^{2}$. Women aged over 40 years were exclud- ed. Hormonal profiles, response to stimulation, and reproductive outcome were reviewed from patients' records. The serum basal FSH level was measured on the second or third day of menstruation and the serum $E_{2}$ level was measured on $\mathrm{hCG}$ administration day in all patients.

\section{Treatment protocol}

The stimulation consisted of a single dose of 100 or $150 \mu \mathrm{g}$ corifollitropin alfa administered on day 2 or 3 of the treatment cycle, depending on patient weight as per the manufacturer's instructions (Elonva, N.V. Organon, Ravensburg, Germany). Patients with an earlier poor ovarian response to stimulation and expected low responders were treated with a starting dose of $150 \mu \mathrm{g}$ corifollitropin alfa, regardless of their weight. Five days later (day 7 or 8 of the cycle), administration of a $\mathrm{GnRH}$ antagonist (Orgalutran, Schering-Plough, France or (etrotide, Merck-Serono SA, USA) at a daily dosage of 0.25 mg was initiated until the day of oocyte retrieval. On day 8 of the stimulation, daily dose of rFSH (Gonal F, Merck-Serono SA, USA) or hMG (Merional, Pharmasure Ltd., Lamone, Switzerland) at a dose of 100-300 IU, depending on patient age, serum $E_{2}$ concentration, and ovarian response, was administered until the day before oocyte retrieval.

In the long $\mathrm{GnRH}$ agonist protocol group, pituitary suppression was initiated with a GnRH agonist (Lorelin, Dongkook CO., LTD, Jincheon, Korea) from the mid-luteal phase of the previous cycle to the day of hCG administration. After pituitary down-regulation, the Lorelin dose was reduced and recombinant FSH (Gonal-F, Merck-Serono SA, USA) was started on the second or third day of the menstrual cycle. On day 2 to 3 of the menstrual cycle, blood samples were obtained by venipuncture for assays of basal FSH level.

To induce final oocyte maturation in both protocols, a recombinant hCG dose (rhCG, Ovidrel, Merck Serono SA, Geneva, Switzerland) of $250 \mu \mathrm{g}$ was given as soon as two or three follicles $>17 \mathrm{~mm}$ were present on ultrasound scan. Transvaginal ultrasound-guided oocyte retrieval was performed 36 hours after hCG injection.

\section{IVF/ICSI}

Retrieved oocytes were cultured in G-IVF medium (Vitrolife, Kungsbacka, Sweden) for 4-6 hours until insemination. In the cases of ICSI, after the cumulus-corona cells were removed by hyaluronidase (SAGE, Pasadena, USA) and mechanically aspirated with stepwise denuding pipettes for no more than 1 minute, the oocyte maturational profile was checked. Denuded oocytes were classified according to meiotic maturity as germinal vesicle (GV, characterized by the presence of this defining structure), metaphase I ( $M l$, characterized by the absence of both a GV and an extruded first polar body), or metaphase II (MII, characterized by the presence of an extruded polar 
body in the perivitelline space). Immature oocytes (MI) were incubated in culture medium at $39^{\circ} \mathrm{C}$ with a humidified atmosphere of $6 \%$ $\mathrm{CO}_{2}$. No hormones were added. Polar body extrusion was checked prior to ICSI. Ml oocytes that extruded their polar body were named "rescued in vitro matured MII oocytes" (MI-MII). Fragmented or shrunken oocytes were classified as degenerated. ICSI was performed on mature oocytes at oocyte denudation (MIl oocytes) and on oocytes that extruded the first polar body between oocyte denudation and ICSI (MI-MII oocytes).

Semen samples obtained by ejaculation in the morning of the oocyte retrieval day were liquefied at $37^{\circ} \mathrm{C}$ for 30 minutes and centrifuged with SpermGrad (Vitrolife) made of two gradient (45\%/90\%) at 2,000 rpm for 5 minutes. After removal of supernatant, $2 \mathrm{~mL}$ of Ham's F-10 medium containing 0.4\% BSA was layered over the sperm pellet prior to being centrifuged at 2,000 rpm for 5 minutes. After washing and swim-up procedures, the sperm in the supernatant were aspirated and used for insemination. The injected oocytes were cultured in G-1 v5 (Vitrolife) and overlaid with $6 \mathrm{~mL}$ of mineral oil (Sigma, USA) in Falcon 1007 culture dishes at $39^{\circ} \mathrm{C}$ under $6 \% \mathrm{CO}_{2}$.

Normal fertilization was assessed on the first day after insemination with the appearance of two pronuclei. The appearance of only one pronucleus or of more than two pronuclei in the cytoplasm was defined as abnormal fertilization. Embryo development, including blastomere number, size, and regularity, and the presence and percentage of fragmentation, was assessed on the morning of day 3 according to Veeck's classification system [11]. ET was performed on day 3 or day 4. The chemical pregnancy rate was determined by a serial serum $\beta$-hCG level of $>10 \mathrm{mlU} / \mathrm{mL} 12$ days after ET.

\section{Statistical analysis}

Mean values were expressed as the mean \pm standard deviation. Statistical analysis of mean values was performed using Student's $t$ tests. Statistical significance was defined as $p<0.05$. All analyses were performed using SPSS ver. 12.0 (SPSS Inc., Chicago, IL, USA).

\section{Results}

A total of 686 patients were enrolled in this study, between May 2011 and May 2013. The corifollitropin alfa group consisted of 113 initiated cycles corresponding to 110 patients, and the control group consisted of 573 initiated cycles corresponding to 502 patients. Patients' baseline characteristics and stimulation characteristics are presented in Table 1. As shown, there were no significant differences in age (corifollitropin alfa group, $32.8 \pm 3.9$ years vs. control group, $33.1 \pm 3.5$ years) and ovarian reserve markers (basal FSH, 7.66 \pm 2.6 $\mathrm{mlU} / \mathrm{mL}$ vs. $5.2 \pm 2.6 \mathrm{mlU} / \mathrm{mL}$ ). In addition, infertility factors between the two groups were similar (Table 1). In the corifollitropin alfa group,
Table 1. Patient baseline and stimulation protocol characteristics

\begin{tabular}{lccc}
\hline Characteristic & $\begin{array}{c}\text { Corifollitropin } \\
\text { alfa }\end{array}$ & $\begin{array}{c}\text { Long GnRH } \\
\text { agonist }\end{array}$ & $p$-value \\
\hline Patient & 110 & 502 & NA \\
$\quad$ No. of patients & $32.8 \pm 3.9$ & $33.1 \pm 3.5$ & NS \\
Mean age (yr) & $7.66 \pm 2.6$ & $5.2 \pm 2.6$ & NS \\
$\quad$ Mean basal FSH level (mlU/mL) & $19(16.8)$ & $97(16.9)$ & NS \\
Primary infertility cause & $58(51.3)$ & $320(55.8)$ & NS \\
Male factors & $2(1.8)$ & $13(2.3)$ & NS \\
Female factors & $34(30.1)$ & $143(25.0)$ & NS \\
Combined factors & & & \\
Idiopathic & $9.5 \pm 1.5$ & $23.8 \pm 2.4$ & $<0.001$ \\
Stimulation characteristics & $3.5 \pm 1.5$ & NA & NA \\
Treatment duration (day) & &
\end{tabular}

Values are presented as mean \pm standard deviation or number (\%). $\mathrm{GnRH}$, gonadotropin-releasing hormone; NA, non-applicable; NS, not significant; $\mathrm{FSH}$, follicle stimulating hormone; $\mathrm{rFSH}$, recombinant follicle stimulating hormone.

the duration of total treatment and additional rFSH was $9.5 \pm 1.5$ days and $3.5 \pm 1.5$ days, respectively. In the control group, the duration of the total stimulation was $23.8 \pm 2.4$ days.

Data regarding responses to stimulation are presented in Table 2 . The two groups did not show differences in endometrial thickness when checked on the hCG administration day. However, the mean $E_{2}$ levels on the hCG administration day were lower in the corifollitropin alfa group than in the control group, an apparent difference that did not reach statistical significance. In the corifollitropin alfa group and in the control group, 1,480 (mean, 13.1 \pm 8.0 ) and 7,636 (mean, $13.4 \pm 8.6)$ oocytes were retrieved, respectively. Among them, 37 (2.5\%) vs. 232 (3.0\%) were MI-MII and 1,248 (84.3\%) vs. 6,269 (82.1\%) were MII oocytes at the time of cumulus removal in the corifollitropin alfa and control groups, respectively. Thus, the maturation rate of the retrieved oocytes was slightly higher in the corifollitropin alfa group than in the control group. However, there were no statistically significant differences between the two groups. When comparing the two groups in terms of good oocytes, fertilized oocytes, and grade I or II embryos, a similar trend was also observed. Seven patients (6.2\%) in the corifollitropin alfa group and 47 patients $(8.2 \%)$ in the control group had ET cycle cancellations due to insufficient ovarian response (lack of follicular development and cryopreservation of all embryos according to monofollicular recruitment, $3.5 \%$ vs. $4.7 \%$ ) or risk of OHSS (2.7\% vs. 3.5\%). There was no significant difference in the ET cycle cancellation rate between the groups. The mean number of fresh embryos transferred for both treatment groups was $2.8 \pm 0.7$ in the corifollitropin alfa group and $3.0 \pm 0.7$ in the control group. The chemical pregnancy rates in both treatment groups were slightly higher in the corifollitropin alfa group $(50 / 106,47.2 \%)$ than in the 
Table 2. Controlled ovarian stimulation results and IVF/ICSI outcomes in two treatment groups

\begin{tabular}{lccc}
\hline & Corifollitropin alfa & Long GnRH agonist & $p$-value \\
\hline No. of cycles initiated & 113 & 573 & NA \\
Endometrial thickness $(\mathrm{mm})$ & $9.7 \pm 0.9$ & $9.5 \pm 1.3$ & $\mathrm{NS}$ \\
Mean of $\mathrm{E}_{2}$ on day of $\mathrm{hCG}(\mathrm{pg} / \mathrm{mL})$ & $3,284.8$ & $4,922.4$ & $\mathrm{NS}$ \\
No. of retrieved oocytes & $1,480(13.1 \pm 8.0)$ & $7,636(13.4 \pm 8.6)$ & $\mathrm{NS}$ \\
Degenerated (\%) & $5(0.3)$ & $28(0.4)$ & $\mathrm{NS}$ \\
GV (\%) & $90(6.1)$ & $616(8.1)$ & $\mathrm{NS}$ \\
MI (\%) & $100(6.8)$ & $489(6.4)$ & $\mathrm{NS}$ \\
MI-MIl (\%) & $37(2.5)$ & $232(3.0)$ & $\mathrm{NS}$ \\
MII (\%) & $1,248(84.3)$ & $6,269(82.1)$ & $\mathrm{NS}$ \\
No. of good oocytes (\%) & $1,285 / 1,480(86.8)$ & $6,501 / 7,636(85.1)$ & $\mathrm{NS}$ \\
No. of fertilized oocytes (\%) & $1,082 / 1,285(84.2)$ & $5,445 / 6,555(83.1)$ & $\mathrm{NS}$ \\
Total no. of embryos (mean) & $1,071(9.7 \pm 6.5)$ & $5,354(9.5 \pm 6.5)$ & $\mathrm{NS}$ \\
No. of good quality embryos (\%, mean) & $668 / 1071(62.4,5.9 \pm 4.7)$ & $3,231 / 5,354(60.3,5.7 \pm 4.7)$ & $\mathrm{NA}$ \\
No. of ET cycles & 106 & 526 & $\mathrm{NS}$ \\
No. of ET cycles cancelled (\%) & $7 / 113(6.2)$ & $47 / 573(8.2)$ & $\mathrm{NS}$ \\
No. of failures related to insufficient ovarian response & $4 / 113(3.5)$ & $27 / 573(4.7)$ & $\mathrm{NS}$ \\
Cases of OHSS & $3 / 113(2.7)$ & $20 / 573(3.5)$ & $\mathrm{NS}$ \\
No. of transferred embryos (mean) & $298(2.8 \pm 0.7)$ & $1,582(3.0 \pm 0.7)$ & $\mathrm{NS}$ \\
Chemical pregnancy rate (\%) & $50 / 106(47.2)$ & $246 / 526(46.8)$ & \\
\hline
\end{tabular}

Values are presented as mean \pm standard deviation or number (\%).

IVF/ICSI, in vitro fertilization-embryo transfer intracytoplasmic sperm injection; GnRH, gonadotropin-releasing hormone; NA, non-applicable; NS, not significant; E2, estradiol; hCG, human chorionic gonadotropin; GV, germinal vesicle; MI, metaphase I; ET, embryo transfer; OHSS, ovarian hyperstimulation syndrome.

control group $(246 / 526,46.8 \%)$, although there was no statistically significant difference.

\section{Discussion}

This study is the first to compare the effects of an antagonist protocol using corifollitropin alfa with the standard COS protocol. Over the past 20 years, the most commonly used COS protocol has been the long $\mathrm{GnRH}$ agonist protocol. The long $\mathrm{GnRH}$ agonist protocol takes approximately three weeks; it effectively prevents premature $\mathrm{LH}$ surges and maintains multifollicular growth. Daily self-injections for approximately three weeks are inconvenient; however, and represent one of the causes of patient stress and financial burdens. Olivius et al. [12] reported that one-third of couples discontinue treatment because of its psychological burden.

The first approach for shortening treatment duration and decreasing the number of injections was the use of the $\mathrm{GnRH}$ antagonist protocol. The use of $\mathrm{GnRH}$ antagonists decreases the duration of $\mathrm{GnRH}$ analog injections by approximately two weeks. Interestingly, Verberg et al. [13] demonstrated a reduced dropout rate in a GnRH antagonist group when compared to a GnRH agonist group, indicating that treatment duration and number of injections is an important factor in determining the risk of dropout.

The second development that can further improve the treatment strategy for IVF is the introduction of corifollitropin alfa. A single injection of corifollitropin alfa, a long-acting rFSH molecule, can replace seven days of daily gonadotropin injections and further decrease the number of injections required in the ovarian stimulation cycle $[3,4,8,14,15]$. In our study, the corifollitropin alfa COS cycle showed similar advantages, such as lower requirements for gonadotropin and shorter durations compared to the long GnRH agonist protocol, proving that it is a more favorable protocol than the standard long GnRH agonist protocol.

In assisted reproduction cycles, the growth of multiple follicles is supported by exogenous gonadotropins. The chance of achieving a pregnancy is related to the number of retrieved oocytes available for IVF/ICSI [16]. Devroey et al. [14] reported that corifollitropin alfa was highly effective in normal ovarian responders, with the number of oocytes retrieved found to be significantly higher compared to individuals injected daily with $\mathrm{rFSH}$, and with similar pregnancy rates. Additionally, the serum $E_{2}$ level on the day of $h C G$ injection was not significantly different between treatment groups $[3,14]$. In the present study, even though there was a lower $E_{2}$ concentration on the day of hCG injection and fewer retrieved oocytes in the corifollitropin alfa group than in the control group, the mean number of matured oocytes in the corifollitropin alfa group tended to be higher than in the control group. These results, although not statistically significant, may be related to the slightly higher chemical pregnancy rate. In ad- 
dition, the ET cancellation rate and risk of OHSS were slightly lower in the corifollitropin alfa group than in the control group. Correspondingly, Polyzos et al. [17] reported that cancellation rates due to low ovarian response in the control group were similar to those of the corifollitropin alfa group. Tarlatzis et al. [9] reported that there was no higher risk for OHSS in infertile women who used corifollitropin alfa. However, the opposite was also reported by Mahmoud Youssef et al. [10], who found that the risk of cancellations was no higher but that the risk of OHSS was five times higher in the corifollitropin alfa group than in the rFSH group, although these differences were not statistically significant. Therefore, further research is needed to determine if corifollitropin alfa is safe in hyper-responders.

In conclusion, although no statistically significant differences were observed between the two groups, the use of corifollitropin alfa seems to offer some advantages to patients undergoing infertility treatment because of its short stimulation duration, safety, lower ET cancellation rate and reduced risk of OHSS.

\section{Conflict of interest}

No potential conflict of interest relevant to this article was reported.

\section{References}

1. Fauser BC, Van Heusden AM. Manipulation of human ovarian function: physiological concepts and clinical consequences. Endocr Rev 1997;18:71-106.

2. Fauser BC, Mannaerts BM, Devroey P, Leader A, Boime I, Baird DT. Advances in recombinant DNA technology: corifollitropin alfa, a hybrid molecule with sustained follicle-stimulating activity and reduced injection frequency. Hum Reprod Update 2009;15:309-21.

3. Devroey P, Fauser BC, Platteau P, Beckers NG, Dhont M, Mannaerts BM. Induction of multiple follicular development by a single dose of long-acting recombinant follicle-Stimulating hormone (FSH-CTP, corifollitropin alfa) for controlled ovarian stimulation before in vitro fertilization. J Clin Endocrinol Metab 2004;89:2062-70.

4. Corifollitropin Alfa Dose-finding Study Group. A randomized dose-response trial of a single injection of corifollitropin alfa to sustain multifollicular growth during controlled ovarian stimulation. Hum Reprod 2008;23:2484-92.

5. Loutradis D, Vlismas A, Drakakis P. Corifollitropin alfa: a novel long-acting recombinant follicle-stimulating hormone agonist for controlled ovarian stimulation. Womens Health (Lond Engl) 2010;6:655-64.

6. Balen AH, Mulders AG, Fauser BC, Schoot BC, Renier MA, Devroey $\mathrm{P}$, et al. Pharmacodynamics of a single low dose of long-act- ing recombinant follicle-stimulating hormone (FSH-carboxy terminal peptide, corifollitropin alfa) in women with World Health Organization group II anovulatory infertility. J Clin Endocrinol Metab 2004;89:6297-304.

7. Bouloux PM, Handelsman DJ, Jockenhovel F, Nieschlag E, Rabinovici J, Frasa WL, et al. First human exposure to FSH-CTP in hypogonadotrophic hypogonadal males. Hum Reprod 2001;16: 1592-7.

8. Duijkers IJ, Klipping C, Boerrigter PJ, Machielsen CS, De Bie JJ, Voortman G. Single dose pharmacokinetics and effects on follicular growth and serum hormones of a long-acting recombinant FSH preparation (FSH-CTP) in healthy pituitary-suppressed females. Hum Reprod 2002;17:1987-93.

9. Tarlatzis BC, Griesinger G, Leader A, Rombauts L, ljzerman-Boon PC, Mannaerts BM. Comparative incidence of ovarian hyperstimulation syndrome following ovarian stimulation with corifollitropin alfa or recombinant FSH. Reprod Biomed Online 2012;24: 410-9.

10. Mahmoud Youssef MA, van Wely M, Aboulfoutouh I, El-Khyat W van der Veen $\mathrm{F}$, Al-Inany $\mathrm{H}$. Is there a place for corifollitropin alfa in IVF/ICSI cycles? A systematic review and meta-analysis. Fertil Steril 2012;97:876-85.

11. Veeck LL. Fertilization and early embryonic development. Curr Opin Obstet Gynecol 1992;4:702-11.

12. Olivius C, Friden B, Borg G, Bergh C. Why do couples discontinue in vitro fertilization treatment? A cohort study. Fertil Steril 2004;81:258-61.

13. Verberg MF, Eijkemans MJ, Heijnen EM, Broekmans FJ, de Klerk C, Fauser BC, et al. Why do couples drop-out from IVF treatment? A prospective cohort study. Hum Reprod 2008;23:2050-5.

14. Devroey P, Boostanfar R, Koper NP, Mannaerts BM, ljzermanBoon PC, Fauser BC, et al. A double-blind, non-inferiority RCT comparing corifollitropin alfa and recombinant FSH during the first seven days of ovarian stimulation using a $\mathrm{GnRH}$ antagonist protocol. Hum Reprod 2009;24:3063-72.

15. Corifollitropin alfa Ensure Study Group. Corifollitropin alfa for ovarian stimulation in IVF: a randomized trial in lower-bodyweight women. Reprod Biomed Online 2010;21:66-76.

16. Hughes EG, Fedorkow DM, Daya S, Sagle MA, Van de Koppel P, Collins JA. The routine use of gonadotropin-releasing hormone agonists prior to in vitro fertilization and gamete intrafallopian transfer: a meta-analysis of randomized controlled trials. Fertil Steril 1992;58:888-96.

17. Polyzos NP, Devos M, Humaidan P, Stoop D, Ortega-Hrepich C, Devroey $\mathrm{P}$, et al. Corifollitropin alfa followed by rFSH in a GnRH antagonist protocol for poor ovarian responder patients: an observational pilot study. Fertil Steril 2013;99:422-6. 